Article

\title{
Genome-Wide Analysis and Expression Profiling of the Heat Shock Factor Gene Family in Phyllostachys edulis during Development and in Response to Abiotic Stresses
}

\author{
Lihua Xie, Xiangyu Li, Dan Hou, Zhanchao Cheng, Jun Liu, Juan Li, Shaohua Mu and Jian Gao * \\ Key Laboratory of Bamboo and Rattan Science and Technology, International Center for Bamboo and Rattan, \\ State Forestry and Grassland Administration, Beijing 100102, China; xielihua0227@163.com (L.X.); \\ leerduo727@163.com (X.L.); hou120314@foxmail.com (D.H.); chengzhan_chao@126.com (Z.C.); \\ liujun_0325@163.com (J.L.); ljgx2003@126.com (J.L.); mush@icbr.ac.cn (S.M.) \\ * Correspondence: gaojian@icbr.ac.cn or gaojianicbr@163.com; Tel.: +86-010-8478-9801
}

Received: 3 December 2018; Accepted: 23 January 2019; Published: 26 January 2019

\begin{abstract}
Heat shock transcription factors (Hsfs) play crucial roles in regulating plant responses to heat and other stresses, as well as in plant development. As the largest monopodial bamboo species in the world, how to adapt to various stresses under the background of global climate change is very important for the sustainable development of bamboo forest. However, our understanding of the function of Hsfs in moso bamboo (Phyllostachys edulis) is limited. In this study, a total of 22 non-redundant Hsf genes were identified in the moso bamboo genome. Structural characteristics and phylogenetic analysis revealed that members of the PheHsf family can be clustered into three classes (A, B and C). Furthermore, PheHsfs promoters contained a number of stress-, hormoneand development-related cis-acting elements. Transcriptome analysis indicated that most PheHsfs participate in rapid shoot growth and flower development in moso bamboo. Moreover, the expression patterns of all 12 members of class A were analyzed under various stresses (heat, drought, salt and cold treatment) through Figurereal-time quantitative polymerase chain reaction (qRT-PCR). Within the class A PheHsf members, PheHsfA1a was expressed mainly during moso bamboo development. Expression of four PheHsfA4s and one PheHsfA2 (PheHsfA4a-1, PheHsfA4a-2, PheHsfA4d-1, PheHsfA4d-2, and PheHsfA2a-2) was up-regulated in response to various stresses. PheHsfA2a-2, PheHsfA4d-1 and PheHsfA4d-2 were strongly induced respectively by heat, drought and $\mathrm{NaCl}$ stress. Through co-expression analysis we found that two hub genes PheHsfA4a-2 and PheHsfA4a-1 were involved in a complex protein interaction network. Based on the prediction of protein interaction networks, five PheHsfAs (PheHsfA4a-1, PheHsfA4a-2, PheHsfA4d-1, PheHsfA4d-2, and PheHsfA2a-2) were predicted to play an important role in flower and shoot development and abiotic stress response of moso bamboo. This study provides an overview of the complexity of the PheHsf gene family and a basis for analyzing the functions of PheHsf genes of interest.
\end{abstract}

Keywords: moso bamboo; heat shock factor gene; abiotic stresses; co-expression

\section{Introduction}

Moso bamboo (Phyllostachys edulis (Carrière) J. Houzeau, synonym Phyllostachys heterocycla (Carrière) is a large woody bamboo of high ecological, economical and cultural value in Asia. Under suitable spring conditions, its shoot can grow from 0 to $20 \mathrm{~m}$ in 45-60 days [1]. Moso bamboo forest covers an area of 3.87 million $\mathrm{hm}^{2}$, accounting for up to $70 \%$ of the Chinese bamboo forest area [2,3]. Because of its rapid growth and highly lignified culms, the annual economic value of moso bamboo production, including 
timber and wood production, reaches 184 billion dollars [4]. Moreover, carbon sequestration in moso bamboo is two to four times greater than that of Chinese fir, making it an important global non-timber forest resource [5]. The growth of bamboo is dependent on natural precipitation and is vulnerable to high temperature and drought. Liu et al. [6] has shown that temperatures $>40{ }^{\circ} \mathrm{C}$ and drought for $>10$ days during August result in severe losses in moso bamboo forests. Drought during spring can reduce moso bamboo shoot growth, yield and quality. From July to September, high temperatures and drought affect the sprouting phase of bamboo. These stresses affect the yield and quality of winter shoots, as well as new bamboo yield in the following year and the yield of wood during subsequent years [7]. Climate change has also been associated with more frequent high temperatures and drought, which in turn reduce the ecological and economical value of moso bamboo. Therefore, it is essential to elucidate the molecular mechanisms involved in heat and drought stress responses to improve stress tolerance in moso bamboo.

To survive high temperature and other stresses, plants have evolved a series of defense strategies [8,9]. Heat shock proteins (HSPs) act as molecular chaperones that protect cells against heat and other stress damage by preventing protein aggregation [10,11]. As the terminal components of the stress signal transduction chain, heat shock stress transcription factors (Hsfs) bind to the promoter regions of HSP genes to regulate transcription in response to stress [12,13], particularly heat stress [14]. Hsfs also contain a highly conserved DNA-binding domain (DBD) at their N-terminal and an oligomerization domain (OD or HR-A/B region) composed of two hydrophobic heptad repeats. Based on the amino acids of the HR-A/B region, plant Hsfs are grouped into three main classes (HsfA, HsfB and HsfC) [15]. Certain Hsfs contain a nuclear localization signal (NLS), a nuclear export signal (NES) and an activator motif (AHA). The AHA motif located at the C-terminus in class A Hsfs confers transcriptional activation. Moreover, a repressor domain (RD) that contains the tetrapeptide LFGV occurs at the C-terminal of class B Hsfs.

Recent studies have revealed that plant Hsfs plays important roles in generating responses to heat and other stimuli, as well as in organ development [16]. Class A HsfA1a is regarded as a master regulator and has a unique role in eliciting heat stress responses in tomato (Solanum lycopersicum L.) [17]. HsfA2 is functionally similar to HsfA1 in regulating thermotolerance, as well as serving as a key regulator in osmotic and oxidative stress responses [18-20]. The expression of HsfA3 is induced in Arabidopsis by heat and drought stress, indicating that HsfA3 might play a role in drought and heat stress signaling [21,22]. The thermotolerance of Arabidopsis with hsfA3 T-DNA insertion mutants was decreased [23]. Moreover, the ectopic overexpression of SIHsfA3 increases thermotolerance and salt hypersensitivity in germination in transgenic Arabidopsis [24]. The Arabidopsis mutant athsfa $4 a$ was more sensitive to dehydration. Furthermore, desiccation tolerance was rescued in athsfa $4 a / B n H S F A 4 a$ seeds to similar levels compared with those of Col-0 [25]. Transgenic chrysanthemum overexpressing CmHSFA4 displayed enhanced salinity tolerance partly due to enhanced $\mathrm{Na}^{+} / \mathrm{K}^{+}$ion and ROS homeostasis [26]. Interestingly, in rice, wheat and Sedum Alfredii, HsfA4a possibly confers cadmium tolerance $[27,28]$. In addition, AtHsfA9 plays a role in embryonic development and seed maturation in Arabidopsis [29]. In group B, the majority of HsfBs act as repressors due to the RD region [15]. However, AtHsfB1 act as repressors of the heat shock response under non-heat-stress conditions, but act as positive regulators of heat shock proteins under heat-stress conditions [30]. In group C, FaHsfC1b from Festuca arundinacea confers heat tolerance in Arabidopsis [31].

Although several Hsf family genes in Arabidopsis and other plant species have been characterized, functional analysis of those in moso bamboo has been limited. The completion of the draft genome sequence of moso bamboo has greatly facilitated the identification of Hsf family at the whole-genome level [32].

In this study, we describe the genome-wide identification and analysis of the PheHsf family of moso bamboo for the first time. In addition, expression patterns of PheHsf genes during development, as well as in response to various abiotic stresses, were also investigated. Our results will provide a foundation and valuable information for future functional analysis of PheHsfs. 


\section{Materials and Methods}

\subsection{Database Searches for Hsf Genes in Moso Bamboo and Analyses of Physicochemical Characteristics}

For more accurate identification of Hsf genes in Moso bamboo, multiple database searches were performed according Hou et al. [33]. First, Hsf mRNA sequences of Oryza sativa and Brachypodium distachyon, obtained from NCBI Nucleotide database (https:/ / www.ncbi.nlm.nih.gov /) as query sequences to blast against the moso bamboo genome database. For the filtration step of the blast process, the Hsf genes were obtained by blast bamboo transcriptome using Hsf mRNA from some other species identified as query with loose $e$-value of $<0.00001$. Next, the protein sequences of putative genes obtained from the first step were blast against the NCBI non-redundant protein database with $e$-value of $<0.0000000001$ by Blast2GO to confirm the identification. The putative genes described as Hsf proteins or proteins belonging to Hsf family were kept, and genes described as other family proteins were abandoned. Then the HSF domain (PF00447) of the HSF family were researched in these putative Hsf proteins to reconfirm our data using the Pfam database (http://pfam.sanger.ac.uk/) and Conserved Domains Database (http:/ / www.ncbi.nlm.nih.gov/Structure/cdd/cdd.shtml) with e-values $<0.001$. Only the gene models containing HSF domain were considered to belong to the Hsf family. Finally, the selected Hsfs were further screened using the full-length non-chimeric (FLNC) reads (http:/ / www.forestrylab.org/db/PhePacBio/) [34]. The moso bamboo Hsf genome sequences, coding sequences, protein sequences, and putative novel or mis-annotated Hsf genes were obtained from moso bamboo genome database. The amino acid sequences of Arabidopsis, rice (Oryza sativa), and B. distachyon Hsf proteins were downloaded from Plant Transcription Factor Database v4.0 (PlantTFDB 4.0, http:/ / planttfdb.cbi.pku.edu.cn/) and Heatster (http://www.cibiv.at/services/Hsf/) [35,36]. Molecular weights and theoretical isoelectric point (pI) were determined using ExPASY (http:/ / web. expasy.org/compute_pi/). CELLO v2.5 Server (http:/ / cello.life.nctu.edu.tw /) was used to predict the protein subcellular locations for candidate PheHsfs [37]

\subsection{Phylogenetic Analysis}

Arabidopsis and rice Hsf gene datasets [38] were used to classify the moso bamboo Hsf genes and predict their functional roles. Multiple sequence alignments of full-length Hsf proteins from Arabidopsis, rice, B. distachyon, and moso bamboo were performed using ClustalX 1.83 (http:// www.clustal.org/) and two online programs (Clustal Omega and MUSCLE) [27,39-41]. An unrooted neighbor-joining (NJ) phylogenetic tree was constructed using MEGA7.0 with 1000 bootstrap replicates. Another phylogenetic tree with only the Moso bamboo Hsfs was also constructed using the amino acid sequences according to the same method.

\subsection{Structural and Motif Analyses of PheHsf Genes}

To confirm subgroup designation through phylogenetic analysis, the Gene Structure Display Server (http://gsds.cbi.pku.edu.cn/) was used to illustrate exon-intron organization by aligning every cDNA sequence and its corresponding genomic DNA sequence [42]. The conserved motifs in the candidate PheHsf sequence were defined by MEME version 4.12.0 (http:/ / meme-suite.org/ tools / meme) [43] using the following parameters: number of repetitions = any, maximum number of motifs $=30$, minimum width $\geq 4$, maximum width $\leq 200$, and only motifs with an e-value $<0.01$ were retained for further analysis. The DBD (DNA Binding domain) and HR-A/B domains were identified using Heatster (http:/ / www.cibiv.at/services/Hsf/). NES domains in the PheHsfs were predicted with the NetNES 1.1 server software (http:/ / www.cbs.dtu.dk/services/NetNES/). NLS domains were predicted using cNLS Mapper software (http:/ / nls-mapper.iab.keio.ac.jp/cgi-bin/NLS_Mapper_form. cgi). AHA domains were predicted according to the conserved motif sequence FWxxF/L, F/I/L [16]. 


\subsection{Cis-Regulatory Element Analysis of PheHsf Genes}

The 1500-bp sequence upstream from the initiation codon of each PheHsf gene was obtained from the moso bamboo genome database. These sequences were used to identify cis-acting regulatory elements with the online program Plant CARE (http://bioinformatics.psb.ugent.be/webtools/ plantcare/html/).

\subsection{Plant Material}

After the surface was sterilized with $1 \%$ formaldehyde, the moso bamboo seeds were germinated in Petri dishes (12-cm diameter) lined with filter paper and containing $10 \mathrm{~mL}$ of sterile water. After 4 days, the germinated seedlings were planted in vermiculite and watered with $1 / 2$ Hoagland's nutrient medium weekly (all plants were grown in $16 \mathrm{~h}$ day $/ 8 \mathrm{~h}$ night at $22{ }^{\circ} \mathrm{C}$ ). Two-month-old seedlings were used for abiotic stress treatments. According to Cheng et al. [44], drought and stress was conducted by incubated seedlings with 20\% ( $m / v)$ PEG6000 and $200 \mathrm{mM} \mathrm{NaCl}$, respectively. Heat stress and low temperature treatments were respectively created by placing seedlings in a $42{ }^{\circ} \mathrm{C}$ and $4{ }^{\circ} \mathrm{C}$ lighted growth chamber according to Liu et al. [45]. The control seedlings were grown without any stress treatment. The second or third mature leaves were collected at $0 \mathrm{~h}, 15 \mathrm{~min}, 30 \mathrm{~min}$, $1 \mathrm{~h}, 3 \mathrm{~h}, 6 \mathrm{~h}, 12 \mathrm{~h}$, and $24 \mathrm{~h}$ after abiotic stress treatments. These materials were immediately frozen and stored in liquid nitrogen until total RNA extraction and real-time quantitative polymerase chain reaction (qRT-PCR).

\subsection{RNA Isolation and Relative Expression Level Analysis of PheHsfs}

For the tissue-specific expression analysis, the RPKM (the reads per kilobase of exon model per million mapped reads) values of $P h e H s f s$ were retrieved from transcriptome sequencing of developing flowers (four stages of flowering and leaves) and shoots of moso bamboo (winter shoots, six shoot heights, and culms) [1,46]. RPKM values were used to analyze the relative expression levels of the PheHsf genes. For the flower development samples, four developmental stages (F1: the floral buds begun to form; F2: the floral organs gradually matured; F3: the flowers were in full blossom; and F4: the embryo formation stage) were defined based on the anatomical structure of floral organs by Gao et al. [46]. For F1 and F2, the buds were collected, respectively. For F3 and F4, spikelets were collected, respectively. Leaves collected from non-flowering moso bamboo were defined as CK1. For the shoot development stage sample, four development stages of shoots were defined based on the continuing measurement of bamboo shoot height and anatomical changes by Li et al. [1]. The eight samples according to the four developmental stages of moso bamboo shoots were as follows: $\mathrm{S} 1$ (winter shoots), S2-S5 (0.5 m, $1 \mathrm{~m}, 3 \mathrm{~m}$, and $6 \mathrm{~m}$, early growth period), S6-S7 (9 m and $12 \mathrm{~m}$, late growth), and CK (clum after leaf expansion, mature period). For S1-S7, the shoot tips of different heights were collected, respectively. For CK2, each top internode was cut from the top to $1 / 2$, then each top internode was divided into basal, middle and top. After that, the samples were cut from the tissue located in the top part of the three internodes above and then mixed.

For qRT-PCR, PheHsfs primers were designed using Primer 3.0 (http:/ / primer3.ut.ee/). Primer sequences, amplicon Length, amplification efficiency, and correlation coefficients are listed in Table S1, and their specificity was verified using the online tool Primer-BLAST (https:/ /www.ncbi.nlm. nih.gov/tools/primer-blast/index.cgi) and the melting curves of PCR products. For every primer pair, a standard curve was constructed to calculate the gene-specific PCR efficiency from the 10-fold series dilution of the mix cDNA template. The $\mathrm{R}^{2}$ (correlation coefficients) and slope values can be obtained from the standard curve. The following formula was used to calculate the corresponding

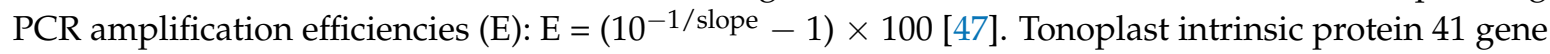
(TIP41) was used as an internal control [48]. The qRT-PCR reactions were conducted using a SYBR Green I master mix (Roche, Mannheim, Germany). The qRT-PCR conditions were as follows: 45 cycles 
of $95{ }^{\circ} \mathrm{C}$ for $10 \mathrm{~s}, 6{ }^{\circ} \mathrm{C}$ for $10 \mathrm{~s}$, and $72{ }^{\circ} \mathrm{C}$ for $20 \mathrm{~s}$. Three replicates were performed for each gene. Gene expression was evaluated using the $2^{-\Delta \Delta \mathrm{Ct}}$ method [49].

\subsection{Co-Expression Network and Protein Interactions of PheHsfs}

The expression correlation of the PheHsfs was calculated by Pearson correlation coefficient (PCC; R-value) using gene expression RPKM values from the high-throughput transcriptome data in $\mathrm{R}$. Expression correlation data were used for the correlation network, and co-expressed gene pairs were filtered with a PCC cut-off of 0.85 as previously described [50]. Cytoscape version 3.4.0 were used to analyze and visualize the network [51].

For the protein interaction networks, the homolog Hsf proteins in rice were constructed by STRING (http:/ /stringdb.org/) using an option value $>0.7$. The homolog proteins of the determined interactive rice proteins were identified in moso bamboo by BLASTP analysis.

\section{Results}

\subsection{The Hsf Family Genes in Moso Bamboo}

To identify Hsf genes in moso bamboo, a total of 41 candidate PheHsf genes were retrieved from the annotation in the Bamboo Genome Database. From these, 19 candidate PheHsf genes with incomplete HSF domains were considered as PheHsf-like genes, which were not selected for subsequent analysis in this study. Twenty-two putative moso bamboo PheHsf genes containing full HSF domains (PF00447) were confirmed by searching the Pfam and the Conserved Domain Databases. However, the CDS sequence for PheHsfA5, PheHsfB4a-1 and PheHsfB4c-1 contained 42 bases (from 268 to 309 base), 102 bases (233 to 336) and 123 base (256 to 377) inserts, respectively, when compared to the cloned cDNA sequence (Fasta S3). For this analysis, the CDS and amino acid sequences of these three genes are based on the CDNA sequences. The CDS and amino acid sequences are listed in supplementary files (Fasta S1 and S2). The identified 22 PheHsf genes were distributed among 22 scaffolds (Table 1). The sequences of 22 PheHsf genes were named according to the corresponding relationship among in rice, $B$. distachyon and moso bamboo. The number of amino acid (aa) sequences of PheHsf proteins ranged from 247 (PheHsfC1b-1) to 679 (PheHsfA1a), the predicted isoelectric points ( $\mathrm{pI}$ ) varied between 4.70 (PheHsfA6a) and 9.81 (PheHsfB4c-1), and the molecular weight (MW) ranged from $26.77 \mathrm{kDa}$ (PheHsfC1b-1) to $75.05 \mathrm{kDa}$ (PheHsfA1a) (Table 1).

\subsection{Phylogenetic Relationships and Multiple Sequence Alignment of PheHsf Genes}

To predict the potential function, an unrooted phylogenetic tree was constructed from an alignment of 94 full length Hsf protein sequences from different species (23 AtHsf, 25 OsHsf, 24 BdHsf, and 22 PheHsf) (Figure 1). Because the alignment results of Hsf proteins in moso bamboo using ClustalX 1.83, MUSCLE, and Clustal Omega were similar, we employed the results of ClustalX 1.83 and MEGA7.0 to illustrate the phylogenetic relationships of the PheHsf family. The PheHsf genes were also grouped into three subgroups, including class A (PheHsfA1a, PheHsfA2a-1, PheHsfA2a-2, PheHsfA4a-1, PheHsfA4a-2, PheHsfA4d-1, PheHsfA4d-2, PheHsfA6b-2, PheHsfA6b-1, PheHsfA6a, PheHsfA5, and PheHsfA7a), class B (PheHsfB1, PheHsfB2a, PheHsfB2c, PheHsfB4c-1, PheHsfB4c-2, PheHsfB4a-2, and PheHsfB4a-1), and class C (PheHsfC1b-1, PheHsfC1b-2, and PheHsfC2b), which is similar to that described in Arabidopsis [52], rice [38], and B. distachyon [53]. In moso bamboo, class A was the largest and consisted of 12 members from six subclasses, however, three subclasses (A3, A8, and A9) in this class were not detected. There were seven members in class B from subclasses B1, B2, and B4, but no members in subclass B3. Class $C$ was the smallest, with only three members. 
Table 1. Overview of PheHsf genes in moso bamboo.

\begin{tabular}{|c|c|c|c|c|c|c|}
\hline Name & Gene ID & Scaffolds & $\begin{array}{c}\text { Number } \\
\text { (aa) }\end{array}$ & $\begin{array}{c}\text { MW } \\
\text { (kDa) }\end{array}$ & PI & $\begin{array}{l}\text { CELLO Localization } \\
\text { (Reliability) }\end{array}$ \\
\hline PheHsfA4a-1 & PH01000018G0140 & РН01000018 & 443 & 49.07 & 5.22 & Nuclear (3.717) \\
\hline PheHsfB2c & PH01000314G0470 & PH01000314 & 382 & 40.12 & 4.86 & $\begin{array}{c}\text { Nuclear (2.907) } \\
\text { Cytoplasmic (1.219) }\end{array}$ \\
\hline PheHsfC1b-1 & PH01000333G0160 & PH01000333 & 247 & 26.77 & 8.71 & $\begin{array}{l}\text { Mitochondrial (1.205) } \\
\text { Chloroplast (1.221) }\end{array}$ \\
\hline PheHsfA1a & PH01000371G0730 & PH01000371 & 679 & 75.05 & 6.06 & Nuclear (2.682) \\
\hline PheHsfB $4 a-1$ & PH01000700G0690 & РН01000700 & 385 & 41.99 & 8.87 & $\begin{array}{l}\text { Cytoplasmic (1.061) } \\
\text { Nuclear (1.481) }\end{array}$ \\
\hline PheHsfC1b-2 & PH01000701G0030 & PH01000701 & 296 & 32.24 & 8.84 & Cytoplasmic (1.557) \\
\hline PheHsfA4a-2 & PH01000746G0370 & PH01000746 & 444 & 49.50 & 5.36 & Nuclear (3.999) \\
\hline PheHsfA4d-1 & PH01000814G0580 & PH01000814 & 554 & 61.26 & 4.95 & Nuclear (2.593) \\
\hline PheHsfC2b & PH01000849G0330 & PH01000849 & 276 & 29.22 & 7.46 & Extracellular (1.087) \\
\hline PheHsfA5 & PH01001018G0530 & PH01001018 & 499 & 55.33 & 5.21 & Nuclear (3.607) \\
\hline PheHsfA7a & PH01001028G0180 & РН01001028 & 381 & 42.51 & 5.94 & Cytoplasmic (1.751) \\
\hline PheHsfB4c-2 & PH01001228G0360 & PH01001228 & 276 & 29.72 & 8.34 & Nuclear (3.293) \\
\hline PheHsfB4c-1 & PH01001554G0080 & PH01001554 & 420 & 45.70 & 9.81 & Nuclear (1.699) \\
\hline PheHsfA4d-2 & PH01002437G0340 & РH01002437 & 453 & 51.34 & 5.18 & Nuclear (4.154) \\
\hline PheHsfB $4 a-2$ & PH01004959G0060 & PH01004959 & 275 & 30.24 & 8.37 & $\begin{array}{c}\text { Chloroplast (1.119) } \\
\text { Nuclear (1.604) }\end{array}$ \\
\hline PheHsfB2a & PH01000000G3800 & РH01000000 & 292 & 31.22 & 4.98 & Nuclear (3.984) \\
\hline PheHsfA6b-2 & PH01000081G0140 & РН01000081 & 362 & 41.44 & 4.97 & Nuclear (3.466) \\
\hline PheHsfA2a-2 & PH01000174G0590 & PH01000174 & 342 & 38.47 & 5.32 & Nuclear (4.565) \\
\hline PheHsfA6a & PH01000194G0800 & PH01000194 & 378 & 42.59 & 4.70 & Nuclear (1.785) \\
\hline PheHsfA6b-1 & PH01000208G0690 & PH01000208 & 348 & 40.04 & 4.89 & $\begin{array}{l}\text { Cytoplasmic (2.207) } \\
\text { Nuclear (2.182) }\end{array}$ \\
\hline PheHsfa2a-1 & PH01003916G0010 & РН01003916 & 358 & 40.31 & 5.49 & Nuclear (3.869) \\
\hline PheHsfB1 & PH01000149G1320 & PH01000149 & 297 & 32.42 & 8.55 & Nuclear (3.609) \\
\hline
\end{tabular}

\subsection{Structure and Motif Analyses of PheHsf Genes}

To better understand the gene structure diversity of PheHsfs, we compared the intron-exon arrangements and the conserved motifs (Figure S1). The number of introns in the Hsf genes of moso bamboo ranged from zero to three. Most of the PheHsfs (19/22) contained one to two introns (Figure S1b). Three introns were found in PheHsf4, whereas none were detected in PheHsfB4a-1 and PheHsfB4c-1.

Based on the known information on functional domains of Hsfs in some model plants [15,54], the sequence and positions of similar domains were identified in the PheHsfs by sequence alignment. As shown in Table 2, five conserved domains (DBD, HR-A/B, NLS, NES, and AHA) were identified. The DBD domain comprised of three $\alpha$-helices $(\alpha 1-3)$ and four $\beta$-sheets $(\beta 1-4)$ were found in all PheHsfs (Figure S2a). HR-A/B domain is critical for one Hsf interacting with other Hsfs to form trimer [15]. All class A PheHsfs have a 21 amino-acid (aa) insertion between HR-A and HR-B regions; class C PheHsfs have seven aa insertions; all class B PheHsfs have no insertion (Figure S2b). NLS and NES domain function in the assembly of a nuclear import complex and the receptor-mediated export in complex with the NES receptor [15]. The majority of the PheHsfs showed the presence of a NES and/or NLS domain. In addition, the activation domain AHA was found in all class A members but not in classes B and C (Table 2). 


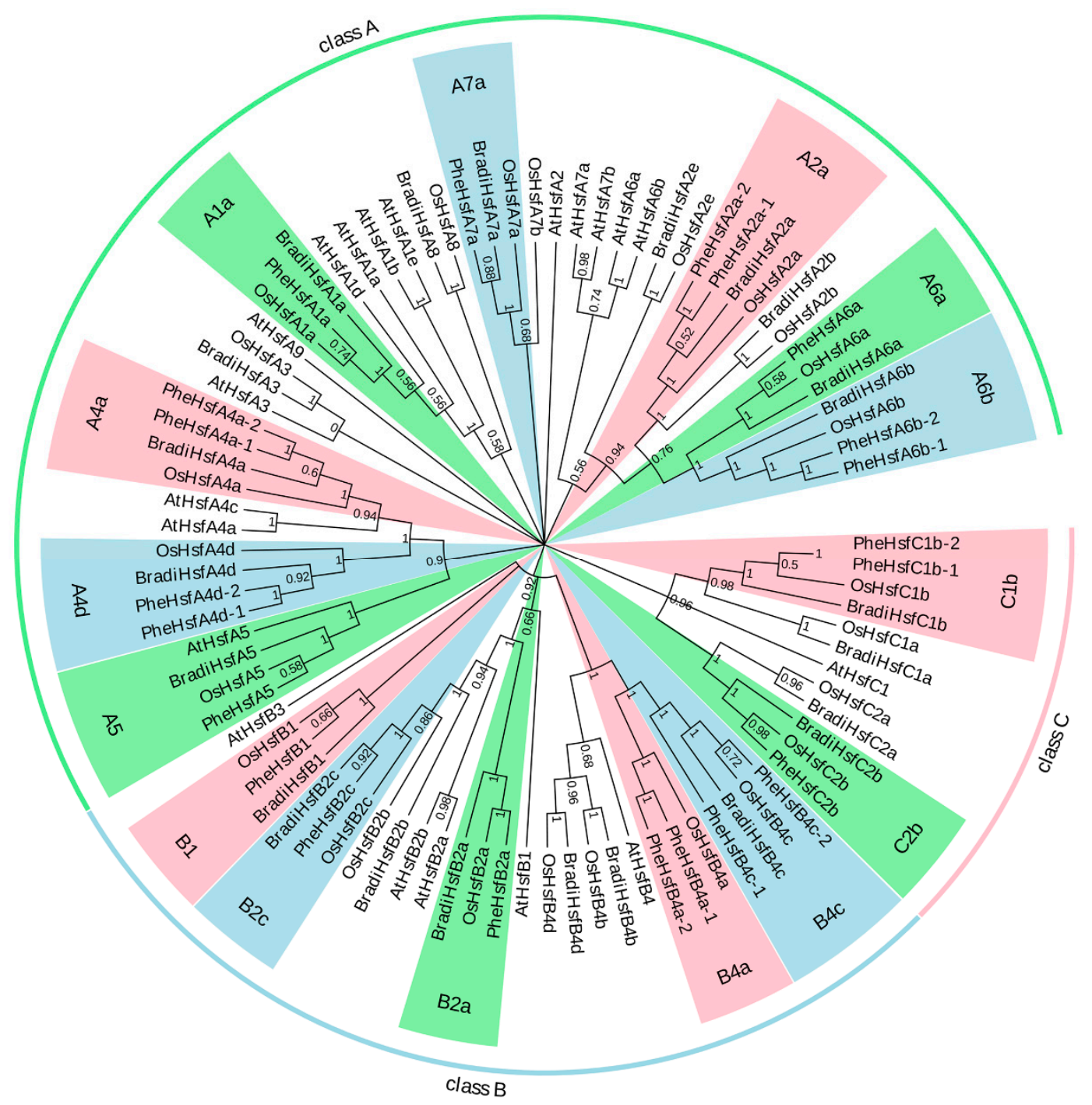

Figure 1. Phylogenetic relationship of PheHsf, AtHsf and OsHsf proteins. Neighbor-joining method and MEGA 7.0 software were used for phylogenetic analysis of Hsf proteins from Phyllostachys edulis (22 PheHsfs), Arabidopsis thaliana (23 AtHsfs), Oryza sativa (25 OsHsfs), and Brachypodium distachyon (24 BdHsfs). The names of subclass are shown outside of the circle. Branch lines of subclass are colored, indicating different Hsf subclasses.

A MEME motif search revealed a total of 19 motifs in the PheHsfs (Figure S1c). Three motifs (1, 2, and 4) constituting the DBD domain were identified. Motif 3 and 5 for the OD domain were detected in class A and C, and motif 6 for the OD domain was observed in class B. Motif 6 and motif 11, motif 8, and motif 17 (NSL) were observed in class A, class B and C, respectively. In general, the structure of the PheHsf proteins is conserved in moso bamboo. Furthermore, motif 7 represented the AHA domain close to the PheHsfA C-terminus (Figure S1c and Table 2). 
Table 2. Functional domains of PheHsfs.

\begin{tabular}{|c|c|c|c|c|c|c|}
\hline Gene & DBD & HR-A/B & NLS & AHA & RD & NES \\
\hline PheHsfA1a & $1-84$ & $121-171$ & (201) ANKKRRLPKQ & (410) SFWEQFLVA & nd & nd \\
\hline PheHsfA2a-1 & $39-128$ & 151-202 & (237) ISKKRRRRID & (314) DDFWEDLLHE & nd & nd \\
\hline PheHsfA2a-2 & $40-129$ & $156-197$ & (238) ISKKRRRRID & (315) DDFWEDLLHE & nd & (165) LLM \\
\hline PheHsfA4a-2 & $14-103$ & $124-172$ & (204) DHHRKKRRLPKPISF & (380) GFWQQFLTE & nd & nd \\
\hline PheHsfA4d-1 & $13-96$ & $112-140$ & (199) FSKKRRAPKI & (363) LFWERFLTE & nd & nd \\
\hline PheHsfA4d-2 & $16-105$ & $120-149$ & (208) FSKKRRVPKI & (382) LFWERFLTE & nd & (252) MELAL \\
\hline PheHsfA5 & $30-134$ & $152-179$ & (241) FQKKRRLTGL & (446) KFWEQFLTE & nd & nd \\
\hline PheHsfA6b-2 & $45-134$ & $163-204$ & (135) KMIKRRRPLS & (318) EDFWEELLNE & nd & nd \\
\hline PheHsfA7a & $74-165$ & 193-227 & (166) KNIKRRRASK & (329) DDVWEELDAI & nd & nd \\
\hline PheHsfB1 & $30-117$ & 176-205 & (253) EDATRKRKRCEEAAARERPFKMIRI & nd & (246) KLFGVLL & nd \\
\hline PheHsfB2a & $7-100$ & $160-183$ & nd & nd & (203) TLFGVTI & (264) LDVLALSL \\
\hline PheHsfB2c & $29-118$ & $199-232$ & nd & nd & (304) RLFGVSI & nd \\
\hline PheHsfB4a-1 & $19-112$ & $110-139$ & (237) RKRLLQEQPPTSPEWKRSMV & nd & (215) KLFGVNL & nd \\
\hline PheHsfB4a-2 & $19-103$ & $201-230$ & (347) RKRSLQEQPPTSPDWKRSMV & nd & (325) KLFGVDL & nd \\
\hline PheHsfC2b & $12-105$ & $140-176$ & nd & nd & nd & (173) LKV \\
\hline
\end{tabular}

DBD: DNA-binding domain; HR-A/B: OD (oligomerisation domain); NLS: Nuclear localization signal; AHA: Activator motifs; RD: Tetrapeptid motif LFGV as core of repressor domain; NES: Nuclear export signal; nd, no motifs detectable by sequence similarity search.

\subsection{Cis-Regulatory Element Analysis in Promoters of PheHsfs}

To predict the biological function of PheHsfs, the $1500 \mathrm{bp}$ upstream sequence from the translation start sites of PheHsf genes were analyzed using the PlantCARE database. The results show that the promoter of each PheHsf has several cis-regulatory elements such as phytohormone- (abscisic acid, jasmonic acid and gibberellic acid), abiotic stress- (low temperature, heat stress, drought, and fungal elicitor), and developmental process-related elements. Figure S3 shows that the ABA-responsive element (ABRE), the MeJA-responsive element (CGTCA-motif), and SA-responsive element (TCA-element) were found in the promoters of 17,16, and 11 PheHsf genes, respectively. The promoters of 12 and 10 PheHsf genes contained the HSE and LTR, respectively. MYB-binding sites involved in drought inducibility (MBS), fungal elicitor-responsive elements (Box-W1) and defense- and stress-responsive elements (TC-rich) were found in 17, 15 and 8 PheHsf genes, respectively. Additionally, meristem expression (CAT-box), meristem-specific activation (CCGTCC-box), and endosperm expression (Skn-1_motif) motifs were found in the 13, 10 and 18 PheHsf genes, respectively. These findings indicate that PheHsfs might be associated with different transcriptional regulatory mechanisms for developmental, hormone and stress processes.

\subsection{Expression Pattern of the PheHsf Genes in Shoot and Flower Development}

Based on the RNA-Seq data of different flowering developmental stages [43] and the internodes of shoots at different heights [1], a heat map was constructed according to the RPKM of 22 PheHsfs (Figure 2). During four flowering developmental stages of moso bamboo, PheHsf genes could be classified into four groups (A, B, C, and D) according to their relative expression levels (Figure 2a). Most of the PheHsfs were highly expressed (RPKM > 10) in at least one stage, and only four PheHsf genes were expressed at low levels $(\mathrm{RPKM}<2)$ in at least two stages during floral development (Table S2). Four members (PheHsfB4a-1, PheHsf15, PheHsf12, and PheHsfB4c-1) of group C had higher transcript accumulation during two earlier stages (F1 and F2) and were downregulated at two later stages (F3 and F4 stage). Group D consisted of 10 PheHsf genes (PheHsfA4a-1, PheHsfB2c, PheHsfA1a, PheHsfA4a-2, PheHsfC2b, PheHsfA7a, PheHsfB2a, PheHsfA2a-2, PheHsfA6b-1, and PheHsfB1), and their expression levels steadily increased at the F3 and F4 stages. Group B comprised of six PheHsfs (PheHsfA4d-1, PheHsfA4d-2, PheHsfA5, PheHsfA6b-2, PheHsfA6a, and PheHsfA2a-1), showing higher transcript accumulation at two later stages (F3 and F4) and in the leaves. Group A only had two genes, PheHsfC1b-1 and PheHsfC1b-2, with expression levels six times and three times higher in leaves than the four stages of floral development, respectively. During shoot growth, Most PheHsfs (19/22) 
showed very low expression levels $(\mathrm{RPKM}<8)$ at all seven stages, and only two genes $($ PheHsfA1a and PheHsfA6b-2) had higher expression levels (RPKM > 20) in at least one stage of bamboo shoot growth (Table S3). Based on the expression profiles, the PheHsfs were classified into four groups (Figure 2b). Among these, PheHsfA1a and PheHsfA2a-2 were clustered into the same group with continuous down-regulated expression from the S1 to S7 stage. However, PheHsfA6b-2 and PheHsfB2c were clustered in the same group, which showed twin peaks during the S2 and S5 stage, respectively.

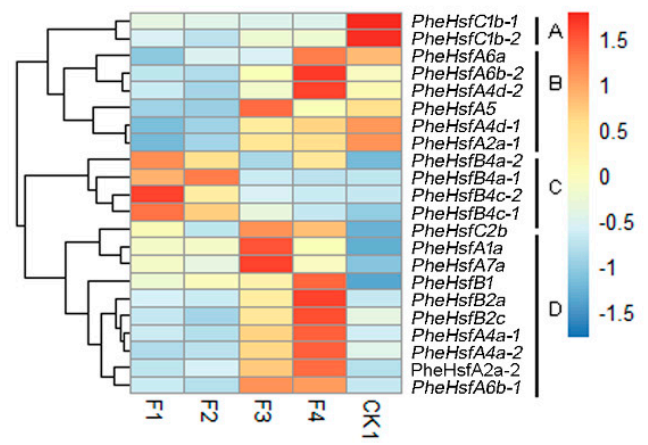

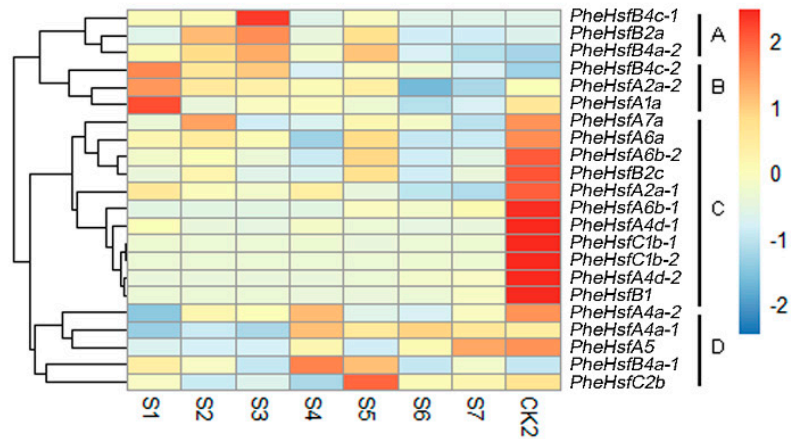

Figure 2. Expression pattern of PheHsfs in developmental flowers and shoots of moso bamboo. (a) The expression profile of PheHsfs in different stages of flowering. F1-F4: The four stages of developmental flowers (the floral buds begun to form, the floral organs gradually matured but did not undergo flowering, the flowers were in full blossom, and the embryo formation stage); CK1: leaves. (b) The expression profile of PheHsfs in different stages of shoots. S1: winter shoot; S2-S7: different heights of shoots $(0.5 \mathrm{~m}, 1 \mathrm{~m}, 3 \mathrm{~m}, 6 \mathrm{~m}, 9 \mathrm{~m}$ and $12 \mathrm{~m})$, and CK2 (culms after leaf expansion). All the samples had one repeat. The heatmap was pictured using $R$ based on the RPKM of Phehsfs in each sample. Details of the RPKM are shown in Tables S2 and S3.

For different periods of flower and shoot development in moso bamboo, the PheHsfA1a and PheHsfA6b-2 genes showed high transcript accumulation in all 11 stages, whereas four genes (PheHsfB4a-1, PheHsfA4d-1, PheHsfA7a, and PheHsfB4a-2) exhibited extremely low transcript accumulation at all stages. Moreover, the other 15 PheHsfs depicted significantly higher transcript levels during flower development than during shoot growth. These findings indicate that these genes have different regulatory roles in moso bamboo flower development and shoot growth.

\subsection{PheHsfAs Expression in Moso Bamboo in Response to Various Stresses}

The expression of 12 PheHsfAs were analyzed by qRT-PCR under four abiotic stresses: high temperature, cold, drought and salt (Figure 3). The expression levels of all members of subclass A were upregulated ( $>2$ folds) after at least one treatment (Table S4, Figure 3b). For heat stress, eight PheHsfAs were upregulated ( $>2$ folds). PheHsfA4d-1, PheHsfA2a-2, and PheHsfA6b-1 rapidly responded to high temperatures, showing upregulation up to $\sim$ nine-fold within $0-1 \mathrm{~h}$ of $42{ }^{\circ} \mathrm{C}$ (Table S4) treatment. Among these, PheHsfA2a-2 ( 22-fold higher than the control) was the most strongly induced gene. For cold stress, the expression levels of seven PheHsfAs (PheHsfA4a-1, PheHsfA1a, PheHsfA4a-2, PheHsfA4d-2, PheHsfA4d-2, PheHsfA5 and PheHsfA2a-2) were at least two-fold greater than the control for at least one of the time points. For drought and salt stress, eight PheHsfAs (PheHsfA4a-1, PheHsfA4a-2, PheHsfA4d-1, PheHsfA4d-2, PheHsfA5, PheHsfA7a, and PheHsfA6a) were upregulated. The expression level of PheHsfA4d-2 was 20-fold higher than control after $3 \mathrm{~h}$ of 20\% PEG treatment. The expression level of PheHsfA4d-1 was $~ 70$-fold higher than the control after $3 \mathrm{~h}$ of $200 \mathrm{mM} \mathrm{NaCl}$ treatment (Table S4). Among the PheHsfA genes, five genes (PheHsfA4a-1, PheHsfA4a-2, PheHsfA4d-1, PheHsfA4d-2, and PheHsfA2a-2) were upregulated relative to the control treatment. (Figure 3b). These findings indicate that these genes might play vital roles in different stress response pathways. 
a

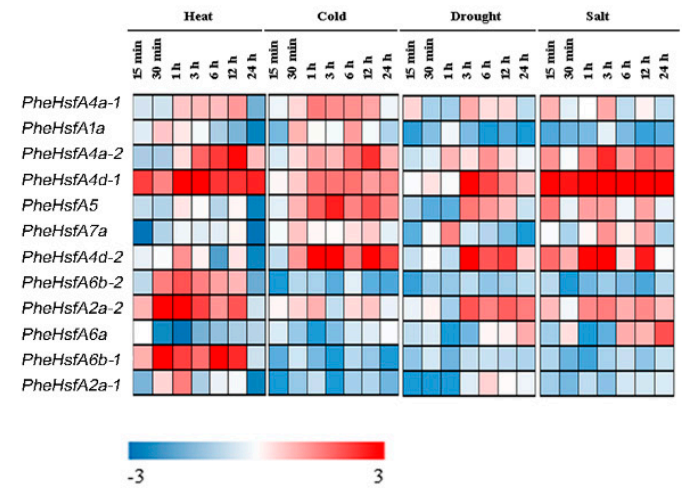

b

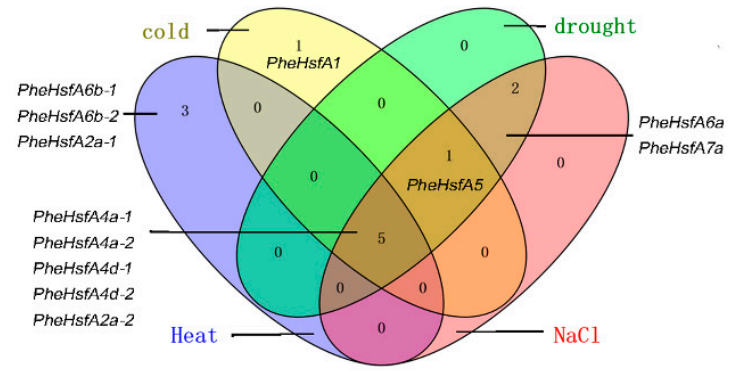

Figure 3. Expression analysis of PheHsfAs under different abiotic stress treatments in moso bamboo. (a) Heat map representation for the expression patterns of PheHsfAs after $15 \mathrm{~min}, 30 \mathrm{~min}, 1 \mathrm{~h}, 3 \mathrm{~h}$, $6 \mathrm{~h}, 12 \mathrm{~h}$, and $24 \mathrm{~h}$ of heat, cold, drought, and salt stresses: expression levels under stress vs. control; the expression results were obtained by qRT-PCR. The different colors correspond to log 2 transformed value. (b) Venn diagram showing the number of overlapping PheHsfAs that are up-regulated > two-fold under abiotic stress: heat, cold, salt, and drought. Details of the expression data are shown in Table S4.

\subsection{Expression Correlation and Interaction Networks}

To further investigate the PheHsf proteins and how they interact with each other, a co-expression network was constructed using expression values of PheHsf genes during shoot and flower development. The connecting gene with PCC magnitude $>0.85$ was recognized as strongly co-expressed genes [36]. The result showed that 18 PheHsf genes and 38 correlations in a co-expression network with PPC $>0.85$ cutoffs were obtained (Figure $4 a$ ). Genes with stronger correlation might play roles as interacting partners in similar biological pathways. Based on the results, PheHsfA4a-1 and PheHsfA4a-2 were recognized as hub genes as nodes with 10 and 9 connectivity in the whole network, respectively, which had more connectivity than other PheHsf genes. Both PheHsfA4a-1 and PheHsfA4a-2 had an up-regulated expression in response to different stress treatments (heat stress, cold, drought and salt). These results indicated that the two PheHsfA4s with a greater role in shoot and flower development also had an important role in stress responses.

To identify the two PheHsfA4a-associated proteins and protein complexes, prediction networks were built with STRING (http://www.string-db.org/) based on the interaction network of rice orthologous genes (Figure 4b). Because the rice orthologous gene of both PheHsfA4a-1 and PheHsfA4a-2 were OsHsfA4a, the identified moso bamboo proteins predicted to participate in the interaction network with PheHsfA4a-1 and PheHsfA4a-2 were the same. They interacted directly with 10 identified proteins, including five HSP70 proteins, one HSP90 protein, and four MAPK proteins. Because PheHsfA2a-2 ( 22-fold higher than the control), PheHsfA4d-2 ( 20-fold higher than the control) and PheHsfA4d-1 ( 79-fold higher than the control) were strongly induced by heat, drought and $\mathrm{NaCl}$ stress, respectively. We also identified the two PheHsfA4d- and PheHsfA2a-2- associated proteins and protein complexes, and PheHsfA4ds and PheHsfA2a-2 were also predicted to interact with HSP70 proteins, HSP90 protein, and MAPK proteins (Figure 4c,d). 
a

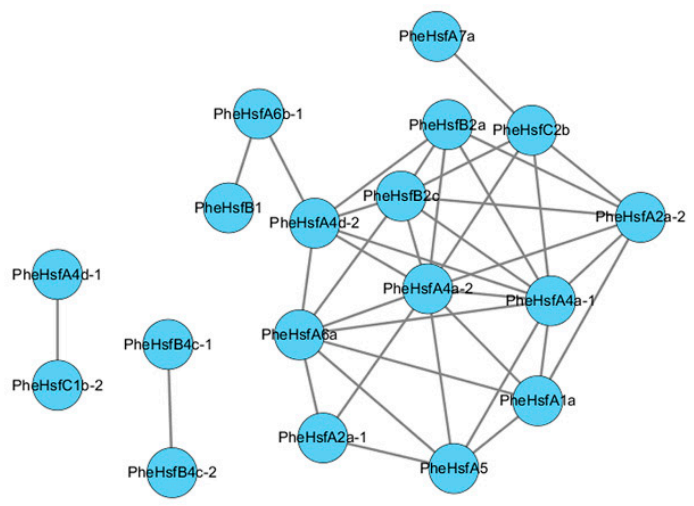

C

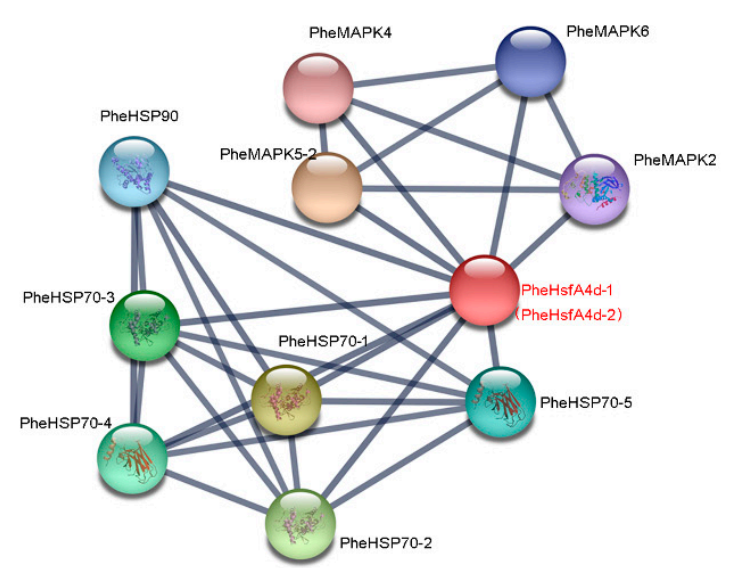

b

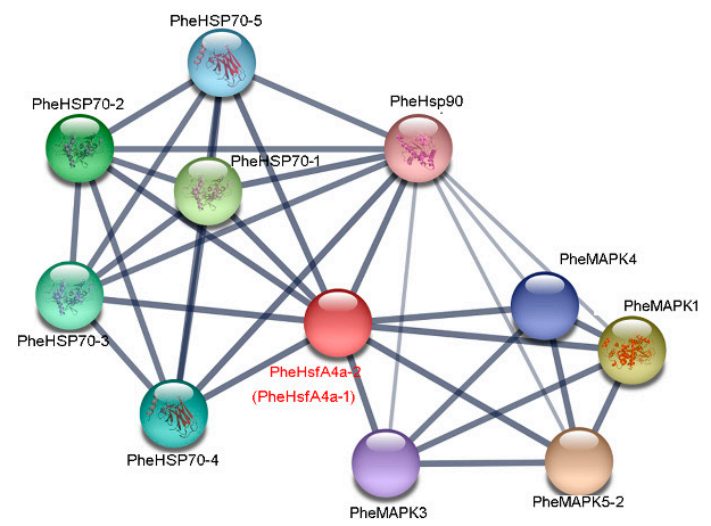

d

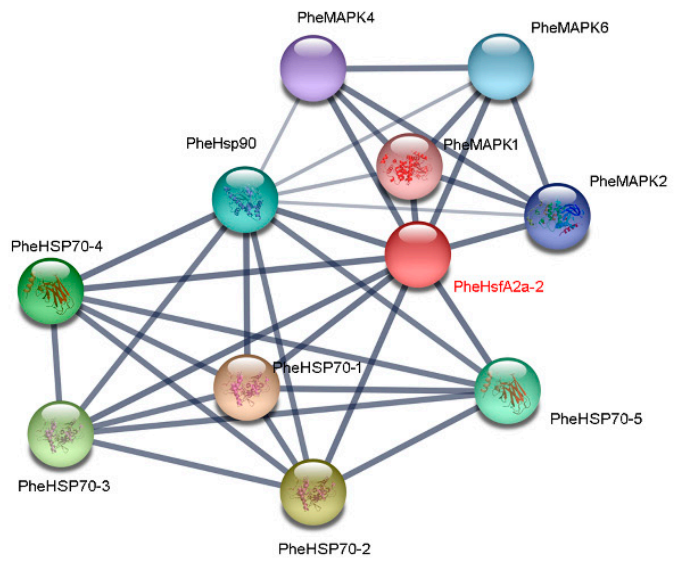

Figure 4. Co-expression network and interaction network of selected PheHsf genes in moso bamboo. (a) The model was built based on RPKM of RNA-seq of shoot and flower development. (b-d) Interaction network of PheHsfA4as, PheHsfA4ds and PheHsfA2a-2 in moso bamboo, respectively. Colored balls (protein nodes) in the network were used as a visual aid to indicate different input proteins and predicted interactors. Protein nodes which are enlarged indicate the availability of 3D protein structure information. Gray lines connect proteins which are associated by recurring textmining evidence. Line thickness indicates the strength of data support.

\section{Discussion}

\subsection{Characterization of the Moso Bamboo Hsf Genes Family}

Hsf genes play essential roles in plant development and in responding to various stress conditions $[55,56]$. To explore the functions of PheHsfs in moso bamboo for the first time, the present study identified a total of 22 PheHsf genes according to the moso bamboo genome database and FLNC reads database $[32,34,57]$.

We found that the moso bamboo has a similar number of Hsfs as rice, B. distachyon, maize, and A. thaliana (22-25). This partially accounts for the support of Hsf conservation during evolution. Phylogenetic analysis of Hsfs in moso bamboo, O. sativa, B. distachyon, and A. thaliana indicated that PheHsfs have a higher degree of sequence similarity with OsHsfs and BdHsfs than AtHsfs, which coincides with the evolutionary relationships among the four species. All three Hsf classes (A, B, and C) were identified in the three monocots and one dicot, implying that the Hsf genes originated prior to the divergence of monocots and dicots. 
In the investigation of conserved Hsf domains and intron-exon structures, all 22 of the PheHsfs contain the necessary (DBD and OD) and/or specific protein domains (NLS, NES, and AHA). The hydrophobic core of DBD domain ensures precise positioning and highly selective interaction with heat stress promoter elements [15]. The OD of plant Hsfs confers distinct patterns of specificity for hetero oligomerization [15]. These AHA motifs, which are located in the C-terminal, are characterized by aromatic (W, F, Y), large hydrophobic (L, I, V) and acidic (E, D) amino acid residues [15]. Those domains might be essential for functional conservation [56]. Twenty of twenty-two PheHsf genes have one intron in their DBD domain (Figure S1), which is an evolutionarily conserved intron [13]. However, no intron was identified in PheHsfB $4 a-1$ and PheHsfB $4 c-1$ of subclass B4, which is different from subclass B4 of rice and B. distachyon [53].

\subsection{Cis-Regulatory Element Analysis in the Promoters of PheHsfs}

Previous studies have illustrated the key roles of Hsfs in developmental processes and stress tolerance through their regulation of target genes [58]. The number and form of cis-elements in the promoter region might play an essential function in the regulation of gene expression in relation to metabolic pathways [59]. The in silico survey of putative cis-elements using PlantCARE showed that 12 of the 22 PheHsfs promoters contained HSEs. This implies that PheHsfs might regulate themselves [55]. Additionally, the promoter region of PheHsfA1a contained more types of development-related cis-elements (Figure S3), which coincides with its constitutive expression during shoot and flower development in moso bamboo (Figure 2).

\subsection{PheHsfAs Involvement in Development Processes}

The 22 PheHsfs exhibited diverse expression patterns during shoot and flower development of moso bamboo under normal conditions. PheHsfA1a was upregulated during shoot and flower development and was constitutively expressed in different tissues (Figure 2). These findings are similar to that in Arabidopsis [60] and Salix suchowensis [61]. Under normal conditions, class A1 Hsfs of Arabidopsis are involved in housekeeping processes, and SsuHsf-A1a of Salix suchowensis are constitutively expressed in different tissues [60,61]. In rice, nearly all class A members showed high expression levels in all tissues $[45,62]$. In this study, nearly all the PheHsfAs (except PheHsfA7a) were found to show high transcription levels in the leaves, culms, and the four stages of flower development, similar to that in rice. Only two members of PheHsfAs (PheHsfA1a and PheHsfA6b-2) have high transcription levels in the seven stages of shoot development. This indicated that the function of PheHsfAs is conserved and/or specific in regulating flower development and shoot growth in moso bamboo.

\subsection{PheHsfAs are Involved in Stress Responses}

Under heat or other stress conditions, plant Hsfs regulate the transcription of target genes (Hsps and other stress-inducible genes) to enhance stress resistance. Recent genome-wide expression profile analyses showed that most of the Hsf genes are upregulated after heat, cold, drought, and salt stress $[56,58,60]$. An increase in the number of Hsf genes has been shown to improve plant stress tolerance [54,63]. In moso bamboo, Zhao et al. identified seven and two PheHsfs that were upregulated after $0.5 \mathrm{~h}$ and $8 \mathrm{~h}$ of high light stress $\left(1200 \mu \mathrm{mol} \cdot \mathrm{m}^{-2} \cdot \mathrm{s}^{-1}\right)$, suggesting that these play vital roles both in response to short-term $(0.5 \mathrm{~h})$ and mid-term $(8 \mathrm{~h})$ high light [64]. However, the regulatory roles of Hsfs in response to other abiotic stresses are unclear. Classes B and C do not harbor AHA motifs, which are essential for the activity of class A Hsfs [16]. Therefore, class A PheHsf genes were selected to further study their response patterns under stress and hormone treatments.

Under heat treatment, 9 of 12 OsHsfAs and 9 of 13 BdHsfAs were upregulated in rice and B. distachyon, respectively [59,62]. In our study, 8 of $12 P h e H s f A s$ were found to be induced by high temperature. Of these, PheHsfA4d-1, PheHsfA2a-2 and PheHsfA6b responded more quickly to heat stress than others. PheHsfA2a-2 showed stronger induced under-heat stress conditions, 
which is similar to that of its homologous genes in rice, Arabidopsis, and B. distachyon $[18,45,53]$. These findings suggest that PheHsfA2a-2 plays an important role in response to heat stress of moso bamboo. However, PheHsfA2a-1, which is the most similar to PheHsfA2a-2 and also belongs to A2a-type PheHsfs, was slightly upregulated $1 \mathrm{~h}$ after heat stress application. PheHsfA4d-2, which is the most similar to PheHsfA4d-1, was less upregulated by heat and salt stress compared to PheHsfA4d-1, but showed higher relative expression levels than PheHsfA4d-1 with cold and drought stresses.

HsfA4a of wheat (Triticum aestivum) and rice conferred cadmium tolerance in yeast and plants, but other Hsfs with similar structure (OsHsfA4d, AtHsfA4a, and AtHsfA4c) did not [27]. HsfA4a of Arabidopsis and chrysanthemum (chrysanthemum cultivar 'Jinba') confers salt and oxidative stress [26,65]. In this study, PheHsfA4a-1, PheHsfA4a-2, PheHsfA4d-1 and PheHsfA4d-2 were upregulated in response to these four abiotic stress (heat, cold, drought and salt). These findings indicate that the three PheHsfA4s could act as potential "nodes" for connecting the above four abiotic stresses.

\subsection{Expression Correlation and Interaction Networks}

In addition, the expression values of PheHsf genes in shoot and flower developmental stages were used to identify potential underlying co-expression networks using Cytoscape. Based on their degree of connectivity, the hub genes PheHsfA4a-1 and PheHsfA4a-2 were identified to play a regulatory role and correlated with other PheHsfs in the complex feedback network. According to the prediction of five PheHsf proteins (four PheHsfA4s and one PheHsfA2a-2) interaction networks, the interactive proteins might include MAPKs and heat shock proteins. MPK5 protein in rice acts as a positive regulator of drought, salt and cold tolerance; is involved in disease resistance and abiotic stress tolerance signaling pathways; and also negatively modulates pathogenesis-related (PR) gene expression and broad-spectrum disease resistance [66]. The MPK1 protein in rice acts downstream of heterotrimeric G protein alpha subunit and small GTPase RAC1 and may regulate the expression of various genes involved in biotic and abiotic stress response [67]. Base on the above information and our results, four PheHsfA4s and one PheHsfA2a-2 may play a very important role in shoot and flower development and stress response.

\section{Conclusions}

In this study, 22 PheHsf genes in moso bamboo were identified for the first time. These genes could be classified into three classes (A, B and C) according to the comparison of the phylogenetic relationships with $O$. sativa, B. distachyon and $A$. thaliana Hsf genes. Expression analyses revealed that two hub genes, PheHsfA4a-1 and PheHsfA4a-2, might act as a potential "node" for crosstalk between developmental processes and abiotic stress responses. Furthermore, PheHsfA2a-2, PheHsfA4d-1 and PheHsfA4d-2 might also act as essential parts in response to stress. These results provide insights into the responses of PheHsfAs to abiotic stresses treatments, although their underlying molecular mechanism requires further study.

Supplementary Materials: The following are available online at http:/ /www.mdpi.com/1999-4907/10/2/100/s1, Figure S1: Phylogenetic tree and gene structure of PheHsf genes, Figure S2: Multiple sequence alignment of the DBD domains and OD region of the PheHsf proteins, Figure S3: Various cis-acting elements in PheHsf genes promoter regions, Table S1: Primer sequences used in gene expression with qRT-PCR experiments, Table S2: The relative level of gene expression of PheHsf genes during developmental flowers (F1 to F4) and leaves (CK)(RPKM), Table S3: The relative level of gene expression of PheHsf genes during growing shoots (S1-S7) and clum (CK)(RPKM), Table S4: Expression analysis of PheHsfAs under different abiotic stress in moso bamboo, Fasta S1: PheHsfs and PheHsfs-like CDS sequence, Fasta S2: PheHsfs and PheHsfs-like protein sequence, Fasta S3: The cloned cDNA sequence of PheHsfA5, PheHsfB4a-1 and PheHsfB4c-1.

Author Contributions: X.L. and J.G. designed the experiments; X.L. and J.L. (Juan Li) performed the tissue and organ collection; L.X. and X.L. performed the experiments; Z.C. formal analysis; J.L. (Jun Liu) and S.M. assisted in equipment maintenance and sample collection; L.X. writing-original draft preparation; X.L. and D.H. writing-review and editing; J.G. review and funding acquisition. L.X. and X.L. contributed equally in this work and should be considered first co-authors. 
Funding: This project was supported by National Keypoint Research and Invention Program in 13th Five-Year (Grant No. 2018YFD0600100).

Conflicts of Interest: The authors declare that they have no conflict of interest.

\section{References}

1. Li, L.; Cheng, Z.; Ma, Y.; Bai, Q.; Li, X.; Cao, Z.; Wu, Z.; Gao, J. The association of hormone signaling genes, transcription, and changes in shoot anatomy during moso bamboo growth. Plant Biotechnol. J. 2017, 16, 72-85. [CrossRef] [PubMed]

2. Lobovikov, M.; Paudel, S.; Piazza, M.; Ren, H.; Wu, J. World Bamboo Resources: A Thematic Study Prepared in the Framework of the Global Forest Resources Assessment 2005; Food and Agriculture Organization of the United Nations: Rome, Italy, 2007; pp. 1-73, 1020-3370.

3. Song, X.; Zhou, G.; Jiang, H.; Yu, S.; Fu, J.; Li, W. Carbon sequestration by Chinese bamboo forests and their ecological benefits: Assessment of potential, problems, and future challenges. Environ. Rev. 2011, 19, 418-428. [CrossRef]

4. Li, L.; Hu, T.; Li, X.; Mu, S.; Cheng, Z.; Ge, W.; Gao, J. Genome-wide analysis of shoot growth-associated alternative splicing in moso bamboo. Mol. Genet. Genom. 2016, 291, 1695-1714. [CrossRef]

5. Zhou, G.M.; Wu, J.S.; Jiang, P.K. Effects of different management models on carbon storage in Phyllostachys pubescens forests. J. Beijing For. Univ. 2006, 28, 51-55. [CrossRef]

6. Liu, L.; Dong, D.; Li, Y.; Li, X.; Bureau, A.M. Investigation of moso bamboo forest under high temperature and drought disaster. World Bamboo Rattan 2014, 12, 24-27. [CrossRef]

7. Zhang, P.; Wang, J.; Zhang, H. Measures of water management and increasing drought resistance of moso forests in Anji County, Zhejiang Province. World Bamboo Rattan 2008, 6, 23-24. [CrossRef]

8. Rizhsky, L.; Liang, H.; Mittler, R. The combined effect of drought stress and heat shock on gene expression in tobacco. Plant Physiol. 2002, 130, 1143-1151. [CrossRef]

9. Chauhan, H.; Khurana, N.; Agarwal, P.; Khurana, P. Heat shock factors in rice (Oryza sativa L.): Genome-wide expression analysis during reproductive development and abiotic stress. Mol. Genet. Genom. 2011, 286, 171-187. [CrossRef]

10. Hartl, F.U.; Hayerhartl, M. Molecular chaperones in the cytosol: From nascent chain to folded protein. Science 2002, 295, 1852-1858. [CrossRef]

11. Queitsch, C.; Hong, S.W.; Vierling, E.; Lindquist, S. Heat shock protein 101 plays a crucial role in thermotolerance in Arabidopsis. Plant Cell 2000, 12, 479-492. [CrossRef]

12. Döring, P.; Treuter, E.; Kistner, C.; Lyck, R.; Chen, A.; Nover, L. The role of AHA motifs in the activator function of tomato heat stress transcription factors HsfA1 and HsfA2. Plant Cell 2000, 12, 265-278. [CrossRef] [PubMed]

13. Pelham, H.R.; Bienz, M. A synthetic heat-shock promoter element confers heat-inducibility on the herpes simplex virus thymidine kinase gene. EMBO J. 1982, 1, 1473-1477. [CrossRef] [PubMed]

14. Von Koskull-Döring, P.; Scharf, K.; Nover, L. The diversity of plant heat stress transcription factors. Trends Plant Sci. 2007, 12, 452-457. [CrossRef] [PubMed]

15. Scharf, K.D.; Berberich, T.; Ebersberger, I.; Nover, L. The plant heat stress transcription factor (Hsf) family: Structure, function and evolution. Biochim. Biophys. Acta 2012, 1819, 104-119. [CrossRef] [PubMed]

16. Kotak, S.; Port, M.; Ganguli, A.; Bicker, F.; von Koskull-Döring, P. Characterization of C-terminal domains of Arabidopsis heat stress transcription factors (Hsfs) and identification of a new signature combination of plant class A Hsfs with AHA and NES motifs essential for activator function and intracellular localization. Plant J. 2004, 39, 98-112. [CrossRef] [PubMed]

17. Mishra, S.K.; Tripp, J.; Winkelhaus, S.; Tschiersch, B.; Theres, K.; Nover, L.; Scharf, K.D. In the complex family of heat stress transcription factors, HsfA1 has a unique role as master regulator of thermotolerance in tomato. Genes Dev. 2002, 16, 1555-1567. [CrossRef] [PubMed]

18. Ogawa, D.; Yamaguchi, K.; Nishiuchi, T. High-level overexpression of the Arabidopsis HsfA2 gene confers not only increased themotolerance but also salt/osmotic stress tolerance and enhanced callus growth. J. Exp. Bot. 2007, 12, 3373-3383. [CrossRef] 
19. Wang, X.; Huang, W.; Liu, J.; Yang, Z.; Huang, B. Molecular regulation and physiological functions of a novel FaHsfA2c cloned from tall fescue conferring plant tolerance to heat stress. Plant Biotechnol. J. 2017, 15, 237-248. [CrossRef]

20. Zhang, L.; Li, Y.; Xing, D.; Gao, C. Characterization of mitochondrial dynamics and subcellular localization of ROS reveal that HsfA2 alleviates oxidative damage caused by heat stress in Arabidopsis. J. Exp. Bot. 2009, 60, 2073-2091. [CrossRef]

21. Sakuma, Y.; Maruyama, K.; Qin, F.; Osakabe, Y.; Shinozaki, K. Yamaguchi-Shino-zaki, K. Dual function of an Arabidopsis transcription factor DREB2A in water-stress-responsive and heat-stress-responsive gene expression. Proc. Natl. Acad. Sci. USA 2006, 103, 18822-18827. [CrossRef]

22. Schramm, F.; Larkindale, J.; Kiehlmann, E.; Ganguli, A.; Englich, G.; Vierling, E.; von Koskull-Döring, P. A cascade of transcription factor DREB2A and heat stress transcription factor HsfA3 regulates the heat stress response of Arabidopsis. Plant J. 2008, 53, 264-274. [CrossRef] [PubMed]

23. Yoshida, T.; Sakuma, Y.; Todaka, D.; Maruyama, K.; Qin, F.; Mizoi, J.; Kidokoro, S.; Fujita, Y.; Shinozaki, K.; Yamaguchi-Shinozaki, K. Functional analysis of an Arabidopsis heat-shock transcription factor HsfA3 in the transcriptional cascade downstream of the DREB2A stress-regulatory system. Biochem. Biophys. Res. Commun. 2008, 368, 515-521. [CrossRef] [PubMed]

24. Li, Z.; Zhang, L.; Wang, A.; Xu, X.; Li, J. Ectopic Overexpression of SlHsfA3, a Heat Stress Transcription Factor from Tomato, Confers Increased Thermotolerance and Salt Hypersensitivity in Germination in Transgenic Arabidopsis. PLoS ONE 2013, 8, e54880. [CrossRef] [PubMed]

25. Lang, S.; Liu, X.; Xue, H.; Li, X.; Wang, X. Functional characterization of BnHSFA4a as a heat shock transcription factor in controlling the re-establishment of desiccation tolerance in seeds. J. Exp. Bot. 2017, 68, 2361-2375. [CrossRef] [PubMed]

26. Li, F.; Zhang, H.; Zhao, H.; Gao, T.; Song, A.; Jiang, J.; Chen, F.; Chen, S. Chrysanthemum CmHSFA4 gene positively regulates salt stress tolerance in transgenic chrysanthemum. Plant Biotechnol. J. 2017, 16, 1311-1321. [CrossRef] [PubMed]

27. Shim, D.; Hwang, J.U.; Lee, J.; Lee, S.; Choi, Y.; An, G.; Martinoia, E.; Lee, Y. Orthologs of the class A4 heat shock transcription factor HsfA4a confer cadmium tolerance in wheat and rice. Plant Cell 2009, 21, 4031-4043. [CrossRef] [PubMed]

28. Chen, S.S.; Jiang, J.; Han, X.J.; Zhang, Y.X.; Zhuo, R.Y. Identification, expression analysis of the Hsf family, and characterization of class A4 in Sedum Alfredii hance under cadmium stress. Int. J. Mol. Sci. 2018, 19, 1216. [CrossRef] [PubMed]

29. Kotak, S.; Vierling, E.; Bäumlein, H.; von Koskull-Döring, P. A novel transcriptional cascade regulating expression of heat stress proteins during seed development of Arabidopsis. Plant Cell 2007, 19, 182-195. [CrossRef]

30. Ikeda, M.; Ohme-Takagi, M. Arabidopsis HsfB1 and HsfB2b act as repressors of the expression of heat-inducible $H s f s$ but positively regulate the acquired thermotolerance. Plant Physiol. 2011, 157, 1243-1254. [CrossRef]

31. Zhuang, L.L.; Cao, W.; Wang, J.; Yu, J.J.; Yang, Z.M.; Huang, B.G. Characterization and functional analysis of FaHsfC1b from Festuca arundinacea conferring heat tolerance in Arabidopsis. Int. J. Mol. Sci. 2018, 19, 2702. [CrossRef]

32. Peng, Z.; Lu, Y.; Li, L.; Zhao, Q.; Feng, Q.; Gao, Z.; Lu, H.; Hu, T.; Yao, N.; Liu, K.; et al. The draft genome of the fast-growing non-timber forest species moso bamboo (Phyllostachys heterocycla). Nat. Genet. 2013, 45, 456-461. [CrossRef] [PubMed]

33. Hou, D.; Cheng, Z.; Xie, L.; Li, X.; Li, J.; Mu, S.; Gao, J. The R2R3MYB Gene Family in Phyllostachys edulis: Genome-Wide Analysis and Identification of Stress or Development-Related R2R3MYBs. Front. Plant Sci. 2018, 9, 738. [CrossRef] [PubMed]

34. Wang, T.; Wang, H.; Cai, D.; Gao, Y.; Zhang, H.; Wang, Y.; Lin, C.; Ma, L.; Gu, L. Comprehensive profiling of rhizome-associated alternative splicing and alternative polyadenylation in moso bamboo (Phyllostachys edulis). Plant J. 2017, 91, 684-699. [CrossRef]

35. Jin, J.; He, K.; Tang, X.; Li, Z.; Lv, L.; Zhao, Y.; Luo, J.; Gao, G. An Arabidopsis transcriptional regulatory map reveals distinct functional and evolutionary features of novel transcription factors. Mol. Biol. Evol. 2015, 32, 1767-1773. [CrossRef] [PubMed] 
36. Jin, J.; Tian, F.; Yang, D.C.; Meng, Y.Q.; Kong, L.; Luo, J.; Gao, G. PlantTFDB 4.0: Toward a central hub for transcription factors and regulatory interactions in plants. Nucleic Acids Res. 2017, 45, 1040-1045. [CrossRef]

37. Yu, C.S.; Lin, C.J.; Hwang, J.K. Predicting subcellular localization of proteins for Gram-negative bacteria by support vector machines based on n-peptide compositions. Protein Sci. 2004, 13, 1402-1406. [CrossRef]

38. Guo, J.; Wu, J.; Ji, Q.; Wang, C.; Luo, L.; Yuan, Y.; Wang, Y.; Wang, J. Genome-wide analysis of heat shock transcription factor families in rice and Arabidopsis. J. Genet. Genom. 2008, 35, 105-118. [CrossRef]

39. Edgar, R.C. MUSCLE: Multiple sequence alignment with high accuracy and high throughput. Nucleic Acids Res. 2004, 32, 1792-1797. [CrossRef]

40. Sievers, F.; Higgins, D.G. Clustal Omega, accurate alignment of very large numbers of sequences. Methods Mol. Biol. 2014, 1079, 105-116. [CrossRef]

41. Thompson, J.D.; Gibson, T.J.; Plewniak, F.; Jeanmougin, F.; Higgins, D.G. The CLUSTAL_X windows interface: Flexible strategies for multiple sequence alignment aided by quality analysis tools. Nucleic Acids Res. 1997, 25, 4876-4882. [CrossRef]

42. Hu, B.; Jin, J.; Guo, A.Y.; Zhang, H.; Luo, J.; Gao, G. GSDS 2.0: An upgraded gene feature visualization server. Bioinformatics 2015, 31, 1296. [CrossRef]

43. Bailey, T.L.; Boden, M.; Buske, F.A.; Frith, M.; Grant, C.E.; Clementi, L.; Ren, J.Y.; Li, W.W.; Noble, W.S. MEME Suite: Tools for motif discovery and searching. Nucleic Acids Res. 2009, 37, W202-W208. [CrossRef]

44. Cheng, Z.C.; Hou, D.; Liu, J.; Li, X.Y.; Xie, L.H.; Ma, Y.J.; Gao, J. Characterization of moso bamboo (Phyllostachys edulis) Dof factors in floral development and abiotic stress responses. Genome 2018, 61, 151-156. [CrossRef]

45. Liu, A.L.; Zou, J.; Zhang, X.W.; Zhou, X.Y.; Wang, W.F.; Xiong, X.Y.; Chen, L.Y.; Chen, X.B. Expression profiles of class A rice heat shock transcription factor genes under abiotic stresses. J. Plant Biol. 2010, 53, 142-149. [CrossRef]

46. Gao, J.; Zhang, Y.; Zhang, C.; Qi, F.; Li, X.; Mu, S.; Peng, Z. Characterization of the floral transcriptome of moso bamboo (Phyllostachys edulis) at different flowering developmental stages by transcriptome sequencing and RNA-Seq analysis. PLoS ONE 2014, 9, e98910. [CrossRef]

47. Bustin, S.A.; Benes, V.; Garson, J.A.; Hellemans, J.; Huggett, J.; Kubista, M.; Mueller, R.; Nolan, T.; Pfaffl, M.W.; Shipley, G.L.; et al. The MIQE guidelines: Minimum information for publication of quantitative real-time PCR experiments. Clin. Chem. 2009, 55, 611-622. [CrossRef]

48. Fan, C.; Ma, J.; Guo, Q.; Li, X.; Wang, H.; Lu, M. Selection of reference genes for quantitative real-time PCR in bamboo (Phyllostachys edulis). PLoS ONE 2013, 8, e56573. [CrossRef]

49. Livak, K.; Schmittgen, T. Analysis of relative gene expression data using real-time quantitative PCR and the $2^{-\triangle \Delta C T}$ Method. Methods 2001, 25, 402-408. [CrossRef]

50. Smita, S.; Katiyar, A.; Chinnusamy, V.; Pandey, D.M.; Bansal, K.C. Transcriptional regulatory network analysis of MYB transcription factor family genes in rice. Front. Plant Sci. 2015, 6, 1157. [CrossRef]

51. Shannon, P.; Markiel, A.; Ozier, O.; Baliga, N.S.; Wang, J.T.; Ramage, D.; Amin, N.; Schwikowski, B.; Ideker, T. Cytoscape: A software environment for integrated models of biomolecular interaction networks. Genome Res. 2003, 13, 2498-2504. [CrossRef]

52. Sultan, S.; Ali, M.; Nawaz, S.; Ali, M.A.; Shahzad, A. Genome wide analysis of heat shock factors (HSF) gene family of Arabidopsis Thaliana. J. Biol. Agric. Healthc. 2016, 6, 69-77.

53. Li, P.S.; Zheng, W.J.; Zhou, Y.B.; Chen, M.; Chai, S.C.; Ma, L.J.; Xu, Z.S. Genome-wide identification, classification and high temperature responses of Hsf family in Brachypodium distachyon. J. China Agric. Univ. 2015, 20, 8-18. [CrossRef]

54. Nover, L.; Bharti, K.; Döring, P.; Mishra, S.K.; Ganguli, A.; Scharf, K. Arabidopsis and the heat stress transcription factor world: How many heat stress transcription factors do we need? Cell Stress Chaperones 2001, 6, 177-189. [CrossRef]

55. Guo, M.; Liu, J.H.; Ma, X.; Luo, D.X.; Gong, Z.H.; Lu, M.H. The plant heat stress transcription factors (HSFs): Structure, regulation, and function in response to abiotic stresses. Front. Plant Sci. 2016, 7, 114. [CrossRef]

56. Xue, G.; Sadat, S.; Drenth, J.; McIntyre, C.L. The heat shock factor family from Triticum aestivum in response to heat and other major abiotic stresses and their role in regulation of heat shock protein genes. J. Exp. Bot. 2014, 65, 539-557. [CrossRef]

57. Zhao, H.; Peng, Z.; Fei, B.; Li, L.; Hu, T.; Gao, Z.; Jiang, Z. BambooGDB: A bamboo genome database with functional annotation and an analysis platform. Database 2014, 2014, bau006. [CrossRef] 
58. Giorno, F.; Guerriero, G.; Baric, S.; Mariani, C. Heat shock transcriptional factors in Malus domestica: Identification, classification and expression analysis. BMC Genom. 2012, 13, 639. [CrossRef]

59. Lescot, M.; Déhais, P.; Thijs, G.; Marchal, K.; Moreau, Y.; Van de Peer, Y.; Rouzé, P.; Rombauts, S. PlantCARE, a database of plant cis-acting regulatory elements and a portal to tools for in silico analysis of promoter sequences. Nucleic Acids Res. 2002, 30, 325-327. [CrossRef]

60. Busch, W.; Wunderlich, M.; Schöffl, F. Identification of novel heat shock factor-dependent genes and biochemical pathways in Arabidopsis thaliana. Plant J. 2010, 41, 1-14. [CrossRef]

61. Zhang, J.; Li, Y.; Jia, H.X.; Li, J.B.; Huang, J.; Lu, M.Z.; Hu, J.J. The heat shock factor gene family in Salix suchowensis: A genome-wide survey and expression profiling during development and abiotic stresses. Front. Plant Sci. 2015, 6, 748. [CrossRef]

62. Mittal, D.; Chakrabarti, S.; Sarkar, A.; Singh, A.; Grover, A. Heat shock factor gene family in rice: Genomic organization and transcript expression profiling in response to high temperature, low temperature and oxidative stresses. Plant Physiol. Biochem. 2009, 47, 785-795. [CrossRef]

63. Liu, A.L.; Zou, J.; Liu, C.F.; Zhou, X.Y.; Zhang, X.W.; Luo, G.Y.; Chen, X.B. Over-expression of OsHsfA7 enhanced salt and drought tolerance in transgenic rice. BMB Rep. 2013, 46, 31-36. [CrossRef]

64. Zhao, H.; Lou, Y.; Sun, H.; Li, L.; Wang, L.; Dong, L.; Gao, Z. Transcriptome and comparative gene expression analysis of Phyllostachys edulis in response to high light. BMC Plant Biol. 2016, 16, 34. [CrossRef]

65. Pérez-Salamó, I.; Szabados, L. The heat shock factor A4A confers salt tolerance and is regulated by oxidative stress and the mitogen-activated protein kinases MPK3 and MPK6. Plant Physiol. 2014, 165, 319-334. [CrossRef]

66. Xie, K.; Chen, J.; Wang, Q.; Yang, Y. Direct phosphorylation and activation of a mitogen-activated protein kinase by a calcium-dependent protein kinase in rice. Plant Cell 2014, 26, 3077-3089. [CrossRef]

67. Shi, B.; Ni, L.; Liu, Y.; Zhang, A.; Tan, M.; Jiang, M. OsDMI3-mediated activation of OsMPK1 regulates the activities of antioxidant enzymes in abscisic acid signalling in rice. Plant Cell Environ. 2014, 37, 341-352. [CrossRef]

(C) 2019 by the authors. Licensee MDPI, Basel, Switzerland. This article is an open access article distributed under the terms and conditions of the Creative Commons Attribution (CC BY) license (http://creativecommons.org/licenses/by/4.0/). 